\title{
Modular Robotics in an African Context
}

\author{
Lund, Henrik Hautop
}

Published in:

Proceedings of IEEE Africon

Publication date:

2011

Link back to DTU Orbit

Citation (APA):

Lund, H. H. (2011). Modular Robotics in an African Context. In Proceedings of IEEE Africon IEEE.

\section{General rights}

Copyright and moral rights for the publications made accessible in the public portal are retained by the authors and/or other copyright owners and it is a condition of accessing publications that users recognise and abide by the legal requirements associated with these rights.

- Users may download and print one copy of any publication from the public portal for the purpose of private study or research.

- You may not further distribute the material or use it for any profit-making activity or commercial gain

- You may freely distribute the URL identifying the publication in the public portal

If you believe that this document breaches copyright please contact us providing details, and we will remove access to the work immediately and investigate your claim. 


\title{
Modular Robotics in an African Context
}

\author{
Henrik Hautop Lund \\ Center for Playware \\ Technical University of Denmark \\ 2800 Kgs. Lyngby, Denmark \\ hhl@playware.dtu.dk
}

\begin{abstract}
In this paper, we review the concept, development and use of modular robotic devices for education, health improvements, and business in Africa. The modular robotics inspired technology has the advantage of allowing any user easy access to a physical construction of new and advanced technology. We conceptualized several educational tools inspired by modular robotics for contextualized IT education in Tanzania, leading to a novel IT degree program and the development of East Africa's first science and business park in Iringa, Tanzania. The prototypes inspired by modular robotics were developed in the local, rural context and tested by local users in hospitals and rehabilitation centres. In this paper, we review the development of both modular building blocks for education and modular robotic tiles for rehabilitation in Tanzania.
\end{abstract}

Keywords-modular robotics, embodied AI, educational robotics, contextualised IT, rehabilitation robotics.

\section{INTRODUCTION}

High tech products of today demand a high degree of technological engineering knowledge to be conceived, designed, developed and tested. If we would like a large proportion of the population to enter into such product development, it is important that we provide tools for education and development that allow people an easy access to the understanding of technology and to the development of technology. This can be viewed as being important especially in regions such as sub-Saharan Africa, where on one hand technology proficiency is low, but on the other hand creativity is high. Indeed, information technology (IT) and engineering can be an agent of change in the context of any developing country, but to achieve that, IT and engineering education needs to be renewed to serve better the needs of the society. The key is the integration of four components:

- shaping the undergraduate IT and engineering education in higher education institutions (HEIs) into a problem oriented approach;

- $\quad$ providing identified, concrete contexts where IT and engineering can make a difference to people's lives;

- providing tools that allow the contextualized creativity to become expressed in engineering solutions, and

- $\quad$ transforming inventions into commercial applications.

This means that an undergraduate IT education starts from teaching the student to identify and elaborate real needs in a particular context (e.g. like a rural hospital), work on this problem, together with a group of other students throughout his/her studies - linking courses to this problem - and finally use the incubation facilities to launch business initiatives based on the learned. Thus, the ultimate goal is to make all the stakeholders - the student and the problem owners - creators of technology, not just its users.

At the same time, it must be realized that students and problems owners in developing regions such as sub-Saharan Africa will not have the same background as students and problems owners in a Western country, so a curriculum and educational technology cannot simply be transferred from a Western country without a high degree of contextualization. For entering into the contextualized IT education and product development based on the problem oriented approach, there is a need for technology tools that allow any user to freely and easily manipulate with the technology hands-on to develop it into products according to the user's own context, problems, and creativity. In this paper, we suggest modular robotic building blocks as a tool for contextualized IT education and product development in developing regions, and show how instantiations of such modular technology have been used for this purpose in Iringa, Tanzania at Tumaini University, Ilembula Hospital, and Neema Crafts rehabilitation unit.

\section{EDUCATIONAL ROBOTICS}

One way of providing students with hands-on experience is through educational robotics based on constructionism. Constructionism was put forward by Seymour Papert [1,2] as a natural extensions of the work by Jean Piaget [3]. Constructionism suggests that learning is achieved most effectively by participation in the construction of artefacts. Piaget suggested and showed that hands-on experimentation is the essential basis for cognitive development, and Vygotsky viewed knowledge as a process, which basically depends on technological and cultural scaffolding [4]. The artefact becomes an "object to think with", which can be used to explore and express ideas which are related to the field (the thing) under investigation. For instance, robots can be used as an educational tool for artificial life and biological investigations, as described in [5], and also we invented and developed the RoboCup Junior competition $[6,7]$ for allowing students to construct their own robots, and learn a lot about real world applications by going through the building process. Indeed, the "real world" testing and experience is of very high importance for the students. For example, Martin suggests that his students run into problems because they tend to build robots that perform properly only under ideal conditions. He states clearly that "Students repeatedly build robots that are not well- 
equipped to deal with the exigencies of the real world, but rather with the specifications of an idealized, abstracted world - a world that the robot designers would like to believe is a close representation of reality, but is not." [8], pp. 189.

For gaining such hands-on, real world experiences, in educational robotics, we have used a three step educational process in which we work with 1) robots with a pre-defined robot body plan, 2) robots with a modifiable robot body plan, and 3) complex robot behaviours (such as team play). By going through this process, the students first get a thorough understanding of robot programming, secondly an understanding of the body and brain relationship (hardware and software relationship), and thirdly an understanding of communication and distributed systems. In such a process, the students are learning 1) to manage the non-deterministic characteristics of the real environment, 2) to integrate hardware and software solutions, and 3) to manage collective and distributed systems [9].

Such educational robotics finds its foundation in embodied artificial intelligence that suggests that there is an intimate relationship between the body (hardware) and the brain (software). To better facilitate this educational robotics approach, we engaged with LEGO in the development of the LEGO Mindstorms that should allow any user to manipulate with both the body and the brain of robots in a fairly easy manner [6, 7], and it has since been used extensively in educational robotics. However, even LEGO Mindstorms impose a certain sequential process and split of building, learning syntax and semantics, programming, debugging, and then finally testing in the real world. Hence, there is a long way from conceiving ideas to actually testing in the real world.

\section{Modular RoBotic BUILDING BLOCKS}

In order to shorten this distance between the conceptions of ideas to the testing in the real world, we conceptualized modular building blocks inspired by modular robotics. We developed examples of the modular concept with inspiration from modern artificial intelligence (e.g. [10]) and modular robotics in order to explore flexibility in activity creation for end-users. The aim is to allow the end-user (e.g. a student, a therapist or a doctor) to utilize her knowledge to adjust the technology in a fast and easy manner. Hence, in contrast to most modular robotics research, here we do not focus on selfreconfiguration but instead focus on user-configuration of modular technology utilizing inspiration and knowledge gained from the development of modular robotics. It is our hypothesis that the inspiration from modular robotics may lead to a highly flexible and easily adjustable system for the end-user. Hence, such a system may be a candidate for the necessary tool for contextualized IT, which was outlined in the Introduction.

As illustrative examples of diversity in such userconfiguration of modular robotic inspired technology, the remix musician Funkstar De Luxe used the approach to compose music and make live performances with technological modules in the form of cubes, cylinders, rolling pins and tiles [11], and therapists made user-configuration of a large subpart of the same user-configurable modular technology for therapy of dementia patients [12].
In modular robotics, each module has a physical expression and is able to process and communicate with its surrounding environment. The communication with the surrounding environment is through communication to neighbouring robotic modules and/or through sensing or actuation. A modular robot is constructed from many robotic modules.

The approach inherits the behaviour-based robotics methods [13] and exploits it with the belief that behaviourbased systems can include not only the coordination of primitive behaviours in terms of control units, but also include coordination of primitive behaviours in terms of physical control units. We, therefore, imagine a physical module being a primitive behaviour. Thereby, the physical organisation of primitive behaviours will (together with the interaction with the environment) decide the overall behaviour of the system. Hence, in a similar way to the control of robot behaviours by the coordination of primitive behaviours, we can imagine the overall behaviour of a (robotic/interactive) artefact to emerge from the coordination of a number of physical (robotic/interactive) modules that each represents a primitive behaviour. In user-configurable modular robotics, it is the user who makes the coordination of the physical modules, e.g. by physically arranging the modules.

We developed such a concept of 'programming by building' [14] as a direct response to our earlier work with LEGO Mindstorms in educational robotics contrasting the Mindstorms split processes of i) building, ii) learning syntax and semantics, iii) programming, iv) debugging, and then finally $v$ ) testing in the real world. With the concept of programming by building, we would investigate 'action in the interaction', where things happen as soon as the user puts two pieces together, and thereby get an immediate feedback (e.g. sound, light, motion) in the construction process, and not only a delayed feedback as when going through the five steps process as known from LEGO Mindstorms development, when the real world feedback is only obtained (late) at step v).

Hence, we developed and used modular tools for exploring the design of flexible and physical components to manipulate conceptual structures. Such modular tools consist of a number of 'intelligent' building blocks that can be manipulated to create both physical functional and conceptual structures. They support our more philosophical claim that both body (physical structure) and brain (functional structure) play a crucial role in intelligence. The focus on building both physical and functional structures suggests that programming of a specific behaviour simply consists of building physical structures known to express that specific behaviour. So, we suggest moving away from programming the artefacts with traditional programming languages, and instead provide methods that allow people to 'program by building' without the need for any a priori knowledge about programming languages. Indeed, we even suggest to completely removing the traditional host computer (e.g. a PC) from the creative process.

Doing programming by building demands the availability of appropriate modular tools, and the limitations of construction of physical and functional expression will be decided by the module design, in terms of physical form, size, material, connectivity, affordance, function, etc. The design of 
optimal modular tools for programming by building is still an open research question. In the following, however, we will describe a few interactive modular tools that are designed based upon modular robotics, following the design principles of modular playware [15]. The tools for physical interaction (e.g. manipulating conceptual structures) in each case consist of a number of 'intelligent' building blocks/modules that each contains processing and communication capabilities. Each module has a physical expression. When attaching more modules together, a user may create a physical structure of modules that process and communicate with each other, depending on how the modules are physically connected to each other. Interaction with the surrounding environment happens through modules that obtain sensory input or produce actuation output. So the overall behaviour of an 'intelligent artefact' created by the users with modules depends on the physical shape of the creation, the processing in the modules, and the interaction between the creation and the surrounding environment (e.g. the users themselves).

\section{MODULES FOR HAND MANIPULATION}

In order to test the concept of programming by building, together with engineering colleagues and students an instantiation of the concept was developed in the form of IBLOCKS $[14,16]$. The first implementation of the I-BLOCKS used an electronic circuit containing a PIC16 microcontroller for processing, and provides four 2-ways serial connections in each I-BLOCK for communication (see Fig. 1). To visualise the concept, it was chosen to make the housing out of rectangular LEGO DUPLO ${ }^{1}$ bricks. Energy power from a $9 \mathrm{~V}$ battery building block was transported through the construction of I-BLOCKS via connectors in the corners on the bottom on each block and connectors in the studs on top of each block.
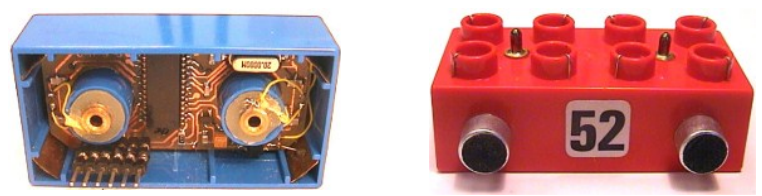

Figure 1. Left: the internals of a building block with micro processor and communication channels. Right: example of input (sensor) building block that contains two microphones.

There exist different types of I-BLOCKS that all share the same standard technology of providing processing and communication capabilities. We term these standard building blocks. In a number of cases, the standard building blocks are extended with the addition of sensors in order to become input building blocks, and in a number of cases extended with the addition of actuation in order to become output building blocks. Input building blocks include building blocks with light sensors, IR sensors, microphones, switches, potentiometer, and output building blocks include building blocks with servo motor, DC motor, IR emitter, LEDs, sound generator, etc. (see examples on Fig. 1).

In order to verify the technological possibilities of the IBLOCKS (e.g. versatility of control methods), we conceived

\footnotetext{
${ }^{1}$ LEGO and LEGO DUPLO are trademarks of LEGO System A/S.
}

different kinds of processing in the I-BLOCKS and had them implemented, making the I-BLOCKS becoming arithmetic blocks, behaviour blocks, language blocks, neural blocks, and spiking neural blocks (e.g. $[14,16])$.

For testing the concept of programming by building and the usability as an educational concept, we (including E. Sutinen, M. Vesisenaho, J. Nielsen, M. Duveskog) collaborated with users from a rural area in Tanzania. Tanzania is an Eastern African country, which has been independent since 1960s. Although Tanzania is economically one of the weakest countries in the world, the demand and the will for development is wide. There are only 7 computers per 1000 people in Tanzania (according to World Bank: ICT at a Glance, 2005), and demand for education and skilled teachers is large. There are about 130 different tribes and many tribal languages. The official languages are Swahili and English, which is the only teaching language in secondary school. Therefore, many pupils may find the gap between primary school and secondary school big, since they have to change from their childhood language to English. In order to facilitate the transfer from primary to secondary school, i.e. from Swahili to English, we introduced the new manipulative technology for language training in a secondary school in a rural area inlands in Southern Tanzania, at Pommern Secondary School about 1-2 hours drive from Iringa (see Fig. 2). For the language training, each I-BLOCK represented an English word (noun, verb, adjective), and the system would give feedback on the grammatical correctness of sentences built with the blocks. Another example included mathematical blocks, so that each block represented a value or a mathematical operator, so the user could explore building mathematical expressions.

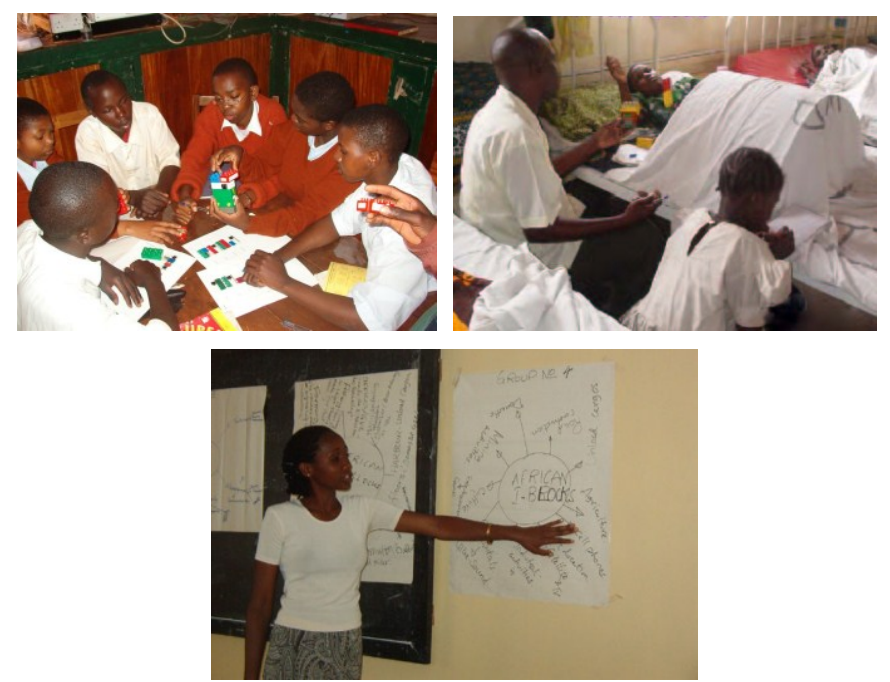

Figure 2. Top: Users from Pommern Secondary School, Tanzania, and user from Ilembula Hospital, Tanzania. Bottom: Developing African I-BLOCKS concept maps together with undergraduate students at Tumaini University, Tanzania.

The educational sessions were structured around the language and mathematics subjects, with the children making language expressions and mathematical expressions with the blocks, thereby testing their own skills. Interestingly, after a very short hands-on free play period with the I-BLOCKS, the children had no problems in understanding the concept of the manipulative technology apart from some power and 
communication problems related to the initial I-BLOCKS prototype. Also, when introducing the I-BLOCKS to undergraduate training at university level at Tumaini University, Iringa, we experienced a surprising confidence and creativity with the technology after only few sessions of handson experiments. The easy access to the new modular manipulative technology prototype prompted us to also investigate the use of the modular tool for simple therapeutic use amongst hospitalised children at the Ilembula Hospital in a rural area of Tanzania (see Fig. 2 right). Also here, the first experiences with similar tasks as for the school children suggested that the modular manipulative technology tool could be beneficial despite the slight problems of the initial prototype. Indeed, together with the undergraduate students at Tumaini University, we explored the use of I-BLOCKS in the Tanzanian cultural context during a full semester course in autumn 2004, and in individual project course activities in spring 2005. The outcome was numerous developments for making the manipulative technology appropriate for the particular cultural context (e.g. see Fig. 2 bottom).

Based upon the feedback, it was evident that most user groups provided feedback related the following problems to be solved: 1) better possibilities for constructing in 3 dimensions, 2 ) better and more reliable power connections, and 3) better and more reliable communication between the blocks. Therefore, a new I-BLOCK prototype with a cubic shape was developed and produced by Nielsen to accommodate "real" 3D structures [16]. This shape is more modular, and gives the possibility of connecting up to 6 other I-BLOCKS to one IBLOCK. Each cubic I-BLOCK used an ATmega128 micro controller, and TL16C754BPN for 4 serial communication ports, allowing for two male connectors and two female connectors on each block. In each of the four corners on each side of the cube, small magnets were placed to ensure that two cubes could lock easily together in 90 degrees rotation intervals. This gave robust and reliable communication and power transfer, as well as simple 3D building possibilities. The final prototype was tested at Ilembula Hospital in Tanzania with hospitalised children (e.g. children with leg fractures who are normally bedridden for 6-8 weeks) in June 2005 (see Fig. 3 ). In this case of hospitalised children and children at an orphanage in a rural area of Tanzania, the children could use the new manipulative technology immediately to make constructions of their own wish without need for instruction or training. It was evaluated by the Tanzanian instructors and nurses to be very effective. Indeed, parents and nurses commented that such a playful modular system had therapeutic benefits, such as that it allowed their children become cheerful and make faster recovery. For instance, one nurse expressed about one child's use of the interactive modules: "She concentrates on this play. If she has other feelings such as a bad feeling, she may forget this", and another nurse that "it gives happiness for the children - in what they have seen and done. Even if they are at home, they will tell the others that at the hospital we did this and this. So the information goes through to others at home."

In general, the project of introducing modular interactive technology was highly recommended by the Tanzanian instructors and the concerned community, especially the children wards of the Ilembula hospital. Quoting one mother “... Mkiendelea kuwepo hapa watoto watapona haraka, kwa sababu wanapata cha kufanya, wanashinda wanacheza na vitu hivi vinavyowavutia..." meaning; "...Your presence speeds recovery of our children because they have something interesting to do rather than staying in bed..."
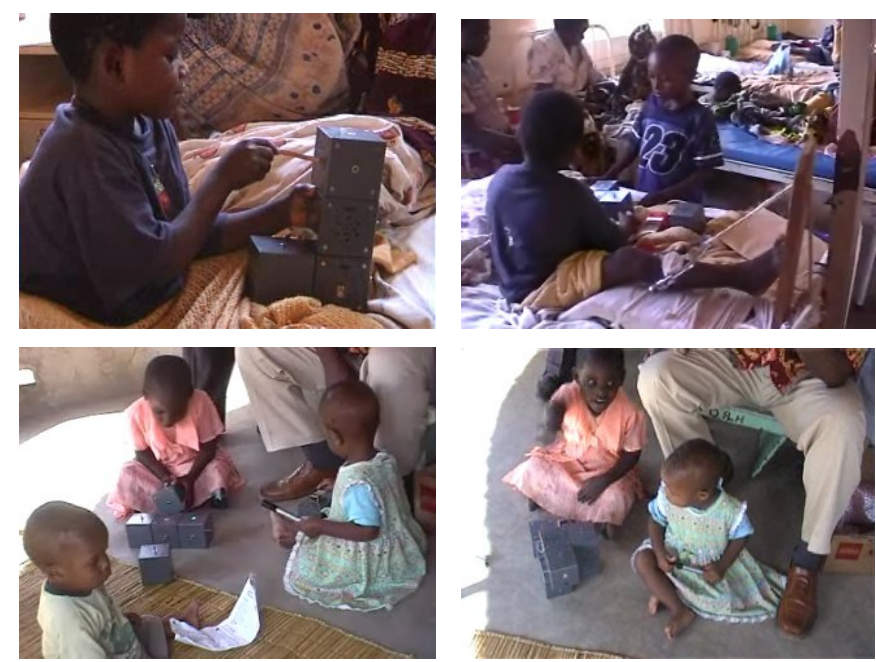

Figure 3. Top: Boys at the children ward of Ilembula Hospital playing with the cubic blocks. Bottom: Girls at the orphanage of Ilembula Hospital building and interacting with the cubic blocks.

\section{MODULES FOR HANDS AND FEET MANIPULATION}

To further explore this kind of use of modular robotics inspired technology for rehabilitation purposes as experienced at Ilembula Hospital, we developed the modular interactive tiles. The system of modular interactive tiles is a distributed system where the modules can attach to each other to form the overall system. The tiles are designed to be flexible and in a motivating way to provide immediate feedback based on the users' physical gaming interaction, following design principles for modular playware [15].
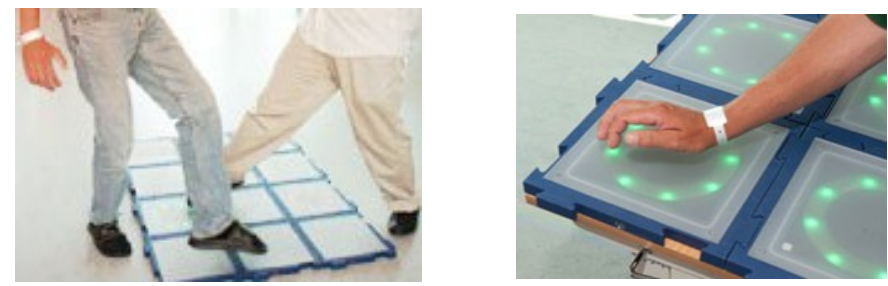

Figure 4. Modular tiles used for feet or hand interaction.

Each modular interactive tile has a quadratic shape measuring $300 \mathrm{~mm} * 300 \mathrm{~mm} * 33 \mathrm{~mm}-$ see Fig. 4 . It is moulded in polyurethane. Inside, the printed circuit board (PCB) has the electronic components mounted, including an ATmega 1280 as the main processor in each tile. At the center of each of the four sides of the quadratic shape infra-red (IR) signals can be emitted and received (from neighboring tiles). On the back of a tile there are four small magnets. The magnets on the back provide opportunity for a tile to be mounted on a magnetic surface (e.g. wall). Each side of a tile is made as a jigsaw puzzle pattern to provide opportunities for the tiles to attach to each other. The cover is made from transparent satinice. 
A force sensitive resistor (FSR) is mounted as a sensor allowing analogue measurement on the force exerted on the top of the cover. On the PCB, a 2 axis accelerometer $(5 \mathrm{G})$ is mounted, e.g. to detect horizontal or vertical placement of the tile. Eight RGB light emitting diodes (LED SMD 1206) are mounted with equal spacing in between each other so they can light up underneath the transparent satinice in a circle.

The modular interactive tiles are individually battery powered and rechargeable. There is a Li-Io polymer battery (rechargeable battery) on top of the PCB. A fully charged modular interactive tile can run continuously for approximately 30 hours and takes 3 hours to recharge - an important long battery life for the utilisation in rural areas in developing countries. The battery status of each of the individual tiles can be seen when switching on each tile and is indicated by white lights. When all eight lights appear the battery is fully charged and when only one white light is lit, the tile needs to be recharged. This is done by turning of the tiles and plugging the intelligent charger into the DC plug next to the on/off switch to recharge each tile.
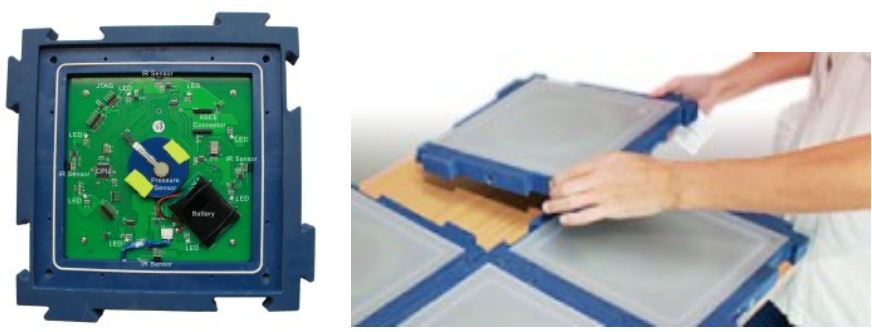

Figure 5. Left: PCB and components of a modular interactive tile. Right: Assembly of the modular interactive tiles as a simple jigsaw puzzle.

On the $\mathrm{PCB}$, there are connectors to mount an $\mathrm{XBee}$ radio communication add-on PCB, including the MaxStream XBee radio communication chip. Hence, there are two types of tiles, those with a radio communication chip (master tiles) and those without (slave tiles). The master tile may communicate with the game selector box (an RFID reader that reads RFID game cards) and initiates the games on the built platform. Every platform has to have at least one master tile if communication is needed e.g. to game selector box or a PC.

With these specifications, a system composed of modular interactive tiles is a fully distributed system, where each tile contains processing (ATmega 1280), own energy source (Li-Io polymer battery), sensors (FSR sensor and 2-axis accelerometer), effectors ( 8 colour LEDs), and communication (IR transceivers, and possibly XBee radio chip). In this respect, each tile is self-contained and can run autonomously. The overall behavior of the system composed of such individual tiles is however a result of the assembly and coordination of all the tiles. The modular interactive tiles can easily be set up on the floor or wall within one minute. The modular interactive tiles can simply attach to each other as a jigsaw puzzle, and there are no wires. The modular interactive tiles can register whether they are placed horizontally or vertically, and by themselves make the software games behave accordingly. Also, the modular interactive tiles can be put together in groups (i.e. tiles islands), and the groups of tiles may communicate with each other wireless (radio). For instance, a game may be running distributed on a group of tiles on the floor and a group of tiles on the wall, demanding the user to interact physically with both the floor and the wall. There are numerous games for both physical and cognitive training.

Work on the modular interactive tiles was performed at the science and business park next to Tumaini University in Iringa, Tanzania to contextualise the modular technology to the local context. Local activity analyses from the science and business park showed a potential for use of highly reconfigurable technological solutions in the area of therapy for children with different needs. Therefore, the science and business park brought the modular tiles to test, development, and use in an orphanage and in the Neema Craft rehabilitation unit for handicapped children over a two years period in 2009-2011. Neema Crafts was started in October 2003 by Susie Hart of the Diocese of Ruaha. Its purpose is to provide handicrafts training and employment for deaf and physically disabled people in Iringa region, Tanzania, and also to change negative attitudes towards people with disabilities in the local community. Local employers are invited to see how skilled the workers at Neema Crafts quickly become when given the opportunity to fulfill their potential. The high quality of their work is changing the attitude of local people towards disabled people [17]. Over 60 people are employed at Neema Crafts, the great majority of them being physically disabled or deaf. The rehabilitation unit of Neema Crafts performs rehabilitation of the physical and mental disabled children of the local region, and the modular interactive tiles were introduced as part of the rehabilitation practice.

An important issue for the rehabilitation practice in this context of the local, rural region is that disabled patients of all kinds are received for rehabilitation sessions. Hence, a rehabilitation tool must be flexible to be adjusted to the needs of the individual patients - needs that vary highly from patient to patient. This was found to be accommodated with the modular tiles on which the local therapists create the activities for the patients modulating exercises and levels simply by (i) changing the physical set-ups with the modular tiles, or (ii) changing the software game or level by swiping an RFID game card over the game selector box. An example of a simple software game found to be effective for several of the patient groups is the "Final Countdown" game, in which each tile is turning off one LED each second, and when the user presses the tile, all LEDs shine up again. If one tile in the platform of tiles goes to zero LEDs turned on, then the user loses the game, i.e. the user has to keep the tiles "alive" by touching them continuously one after another. By changing the size of the platform, the therapist can change the level of the game (a larger platform is more difficult to keep alive than a smaller platform), or the therapist can change the level by using a game card that tells if the countdown time should be faster or slower.

Figure 6 shows a typical session at Neema rehabilitation unit with a 4 years old girl with cerebral palsy with fixed contracture in born limbs. Initially, the girl is supported in the walking on the modular tiles holding hands with the therapist or a caretaker (e.g. mother) while playing the colour race game where she is trying to hit one of the colours on the tiles floor. After a while, the therapist moves the six tiles next to the walking bar, and the child is allowed to perform the game on her own with no help from therapist or caretaker. On figure 7, 
for another child who is less mobile, the therapist simply has made the tiles platform smaller using only 4 tiles instead of 6 tiles, allowing the cripple child to sit on the small tiles platform using his hands. For the therapist, the change to make the rehabilitation activity fit to the individual child is done simply by physically building an appropriate platform with the modules.
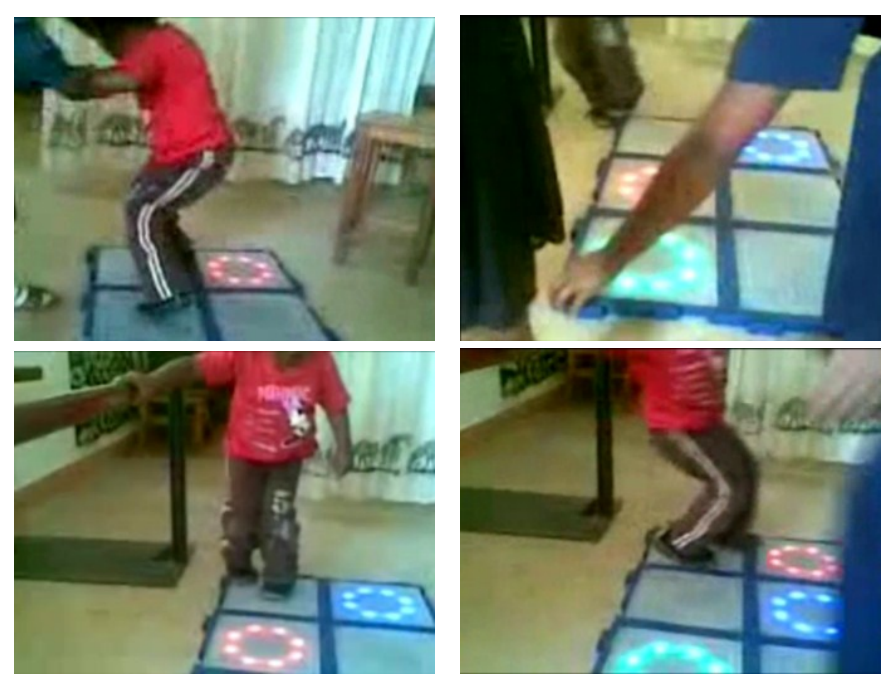

Figure 6. A typical session at Neema rehabilitation unit where the modularity of the tiles is used to change the set-up allowing to gradually change the level of the activity from supportive to autonomous performance of the child.

Physiotherapist Beatrice Mswai of the Neema rehabilitation units evaluates the implementation of the modular interactive tiles technology as follows: "These tiles are the most amazing equipment. Children who are physically challenged seem to enjoy them a lot especially children with cerebral palsy to be specific". Indeed, the children with cerebral palsy perform different exercises on the tiles, which the physiotherapist adjusts to their age and capabilities. The physiotherapist classifies the activities as follows:

- For very young children (age 0-1) they seem to enjoy watching the different colour flashing across their eyes which helps in concentration on certain objects since with this age there is not much activeness.

- For children aged 1-2 years, they also enjoy the flashy colours and they also understand simple games like: I) Reach, II) Colour-race, III) Music colour-race, IV) Stepper. For these children, it is not only about fun, but they understand the rules and they follow them correctly, although they need an adult to lead them sometimes.

- For children aged 3-5 years, they are more excited about the tiles, and the games which they find interesting are: I) Dance floor, II) Final Countdown beat, III) Flash paint, IV) Colour race. They also understand the rules of the game and they can play alone without an adult player instructing them.

- For children aged 5-7 years, at this age they are more cooperative and they enjoy the most complex games like: I) Simon says, II) Paint, III) Music paint, IV) Final countdown. This age group can play in a big group and cooperate very well.

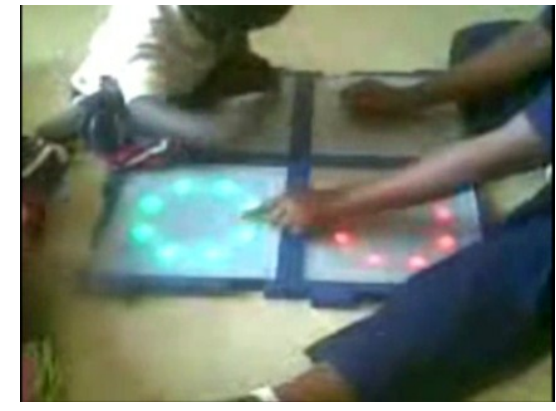

Figure 7. Therapy session with a 7 years old boy who is cripple and with mental disability, playing a game with his hands together with the therapist. As he cannot move much, a small set up of only 4 tiles is used by the therapist.

Physiotherapist Mswai states that "Generally the tiles really help not only in motivating the children but also in an educative way because they learn the colour and how to count which is amazing. They not only use their feet but also sometimes their hands and I find that they like more with the hands and this is because to some of them their conditions do not allow them to stand" (see Fig. 7), and further that "People like care takers, assistants, etc., who work with physically challenged children, are amazed when they see the equipment and how much the children enjoy it."

Interestingly, observations of therapeutic practices with the modular interactive tiles for a variety of other patient groups (cardiac patients, stroke patients and COLD (chronic obstructive lung disease) patients) also suggest that therapists do indeed take advantage of the flexibility that the modular system provides [18]. The therapists create the activities for the patients modulating exercises and levels by changing the physical set-ups with the modular tiles system. This is indeed important in the daily therapeutic practice, which is characterized by the treatment of patients with many different, individual needs, and especially in a context of rural areas in developing countries where patients groups are very diverse and often much more diverse than in specialized units in Western hospitals and rehabilitation centers. A system should therefore provide flexibility to adjust to treatment area, activity, patient level, and patient fatigue. The observations of therapeutic use suggest that the modularity gained from the inspiration from modular robotics is one solution to provide such flexibility [18]. At the same time, the modularity provides an ease of use, possibility of use anywhere, and robustness through distributed processing. Indeed, the modular interactive tiles are easily brought from use at the rehabilitation unit in Iringa out to e.g. orphanage in the local, rural area, where the children simply assemble the modular tiles within minutes and start interacting with them. It becomes possible to perform the exercises in the local context for the local need.

\section{DISCUSSION AND CONCLUSION}

In this paper, we have reviewed the concept, development and use of modular robotic devices for education and health improvements in Africa. One example showed the use of modular robotics for easily manipulating abstract concepts in a hands-on fashion (e.g. for second language and mathematics education), and the second example showed the use of modular robotics for modulating full body interaction (e.g. for 
rehabilitation of cognitive and physically impaired children). Other examples of modular systems for manipulating abstract concepts include the GameBlocks for African users [19], active surfaces for aquatherapy for children with special needs [15], and more recent American systems such as siftables, cubelets, topobo, and Blinky Bots to name a few.

Both examples, which are reviewed here in this paper, highlight the approach of taking inspiration from modular robotic technology to offer a hands-on opportunity for anybody to make contextualized solutions. Indeed, the approach can be viewed as offering a technological approach for participatory design and user-driven innovation. If designed in the right manner, the technological building blocks can become the tool that allows people to perform user-driven innovation of engineering solutions even if people possess little engineering knowledge. By putting the building blocks together, the user is investigating and making new solutions. Being able to do so demands that the technological building blocks are welldesigned for this purpose, and making such design is a challenge, which demands insight into user competencies, interaction design, affordance, engineering, modular robotics, computer science, etc. For instance, it is an interesting challenge to understand if building blocks could and should be similar for all user groups and contexts, or if and how they could/should differ dependent on activities and contexts. Science needs to shed light on how different cultures, different age groups, different genders, etc. understand and interact with technological building blocks in different contexts.

In general, the modular system seems to become an object to think with, and the modularity invites to physical manipulation and reconfiguration. In the process of physical manipulation, the user starts understanding and developing the functionality of the artefact. There is immediately action in the interaction, so the user is not only learning about the abstract thinking that the system may represent, but at the same time, the user is able to try the system in new, innovative configurations. Hence, the system becomes a true engine for innovation allowing the user to creatively invent, build and test new developments as the exploration is happening. Indeed, the examples which are reviewed in this paper show how pupils, students, and professionals are able to develop their own, contextualized solutions by manipulating technological building blocks.

Therefore, we advocate such an approach for the contextualized IT development, which demands (i) a problem oriented educational approach, (ii) providing identified concrete contexts where IT and engineering can make a difference to people's lives, (iii) providing tools that allow the contextualized creativity to become expressed in engineering inventions, and (iv) transforming such inventions into commercial applications. Indeed, technological building blocks can become an integrated solution to the demands (i)-(iv) as a tool that allows the users to engage in a contextualized problem oriented approach, which results in applications of real value in the local community. As such, the general approach may hopefully hold much higher, general potential than the small applications shown in this paper, which are meant only as illustrative examples of the general approach. Indeed, we hope that the general approach may open up for new contextualized
IT education, development and commercial exploitation in Africa and other developing regions, as well as in developed regions of the world.

\section{ACKNOWLEDGMENT}

The author would like to thank collaborators including E. Sutinen, M. Duveskog, M. Vesisenaho, B. Mswai, J. Nielsen, P. Marti, L. Pagliarini, R. Abdalahman. Also, the author would like to thank participating people at Tumaini University, Ilembula Hospital, Pommern Secondary School, and Neema Crafts. Initial I-BLOCKS work was funded by the Danish National Research Council project "Intelligent Artefacts" and the EU FET project "HYDRA".

\section{REFERENCES}

[1] S. Papert. Mindstorms: Children, Computers, and Powerful Ideas. Basic Books, New York, 1980.

[2] S. Papert. Constructionism: A New Opportunity for Elementary Science Education. A proposal to the National Science Foundation, 1986.

[3] J. Piaget and B. Inhelder. La psychologie de L'enfant. Paris, P.U.F, 1966.

[4] L. S. Vygotsky. Thought and language. Cambridge; MIT Press, 1986.

[5] O. Miglino, H. H. Lund, M. Cardaci "Robotics as an Educational Tool" In Journal of Interactive Learning Research, 10:1, 25-48, 1999.

[6] H. H. Lund and L. Pagliarini. "Robot Soccer with LEGO Mindstorms". In Asada (ed.) Proceedings of RoboCup'98, LNAI 1604, SpringerVerlag, Heidelberg, 1999

[7] H. H. Lund, and L. Pagliarini. "RoboCup Jr. with LEGO Mindstorms". In Proceedings of Int. Conf. On Robotics and Automation (ICRA2000), IEEE Press, NJ, 2000

[8] F. Martin "Ideal and Real Systems: A Study of Notions of Control in Undergraduates Who Design Robots". In Y. Kafai and M. Resnick (Eds.), Constructionism in Practice: Rethinking the Roles of Technology in Learning, MIT Press, MA, 1994.

[9] H. H. Lund. "Robot Soccer in Education". Advanced Robotics Journal,13:8, 737-752, 1999.

[10] R. Pfeifer and C. Scheier. Understanding Intelligence. MIT Press, Cambridge, MA, 1999.

[11] H. H. Lund and M. Ottesen "RoboMusic" In Sugisaka and Takaga (eds.) Proceedings of 12th International Symposium on Artificial Life and Robotics (AROB'12), ISAROB, Oita, 2007.

[12] H. H. Lund, P. Marti, A. Derakhshan, R. Beck, T. Klitbo, and J. Nielsen, "Modular Robotics for Novel Tools in Dementia Treatment". In Proceedings of 15th IEEE Intl. Symposium on Robot and Human Interactive Communication (RO-MAN 06), IEEE Press, 655-661, 2006.

[13] R. A. Brooks. "A robust layered control system for a mobile robot". IEEE Journal of Robotics and Automation, 2(1):14--23, 1986.

[14] H. H. Lund "Intelligent Artefacts" In Sugisaka and Tanaka (eds.) Proceedings of 8th International Symposium on Artificial Life and Robotics, I11-I14, ISAROB, Oita, 2003.

[15] H. H. Lund, and P. Marti. "Designing Modular Robotic Playware." In Proc. of 18th IEEE International Symposium on Robot and Human Interactive Communication (Ro-Man 2009), IEEE Press, 2009, 115-121.

[16] J. Nielsen and H. H. Lund "Contextualised Design of African IBLOCKS". In H. H. Lund et al. (eds.) Proceedings of Fourth IEEE International Workshop on Technology for Education in Developing Countries. IEEE Press, 2006: 14-18, 2006.

[17] www.neeamacrafts.com (checked 22/6/2011)

[18] H. H. Lund and C. B. Nielsen "Modularity for Modulating Exercises and Levels - Observations from Cardiac, Stroke, and COLD Patients Therapy", submitted to URAI 2011.

[19] A. C. Smith "Tangible cubes as programming objects". In Proceedings of the $16^{\text {th }}$ International Conference on Artificial Reality and Telexistence (ICAT 2006), IEEE Computer Society, 2006. 